\title{
Status of Knowledge of Chemical Erosion of Carbon and Critical Issues for Extrapolation to ITER
}

\author{
Joachim Roth \\ Max-Planck-Institut für Plasmaphysik, EURATOM Association \\ D-85748 Garching, Germany
}

\begin{abstract}
:
Chemical erosion of-carbon based plasma-facing materials and the re-deposition of tritium containing carbon layers are critical processes for the material choice in ITER. At the occasion of the workshop on Plasma-Surface Interaction related to Fusion (PSIF) the present status of knowledge was defined, suggestions for improved measurements discussed and the importance of atomistic modeling of individual processes underlined. In the present article the status of knowledge for both processes is outlined and important unresolved issues are identified: the extrapolation of measured erosion yields at high particle fluxes towards low energies as expected in detached divertor plasmas and the transport and deposition of hydrocarbon molecules on the divertor plate and walls.
\end{abstract}




\section{Introduction:}

Enhanced erosion of carbon based materials due to chemical interaction with hydrogen ions is an important disadvantage for the proposed use of carbon fiber composites (CFCs) in ITER. Main issues are the lifetime of CFC components, the plasma contamination with carbon ions and the re-deposition of carbon layers together with hydrogen isotopes. The nature of the emitted hydrocarbons and radicals is important in the understanding of carbon transport in fusion devices and determines sticking to vessel walls and tritium inventory.

Chemical erosion has been investigated in great detail for more than 25 years. Total yields were measured as function of parameters such as particle energy, surface temperature and ion flux. The composition of the emitted hydrocarbons and radicals has been determined using mass spectroscopy. In detailed surface physics studies for thermal hydrogen atoms the individual steps of the erosion process, such as hydrogen adsorption, changes in carbon hybridization, and thermal desorption of reaction products were investigated and quantified [1]. An analytic description was developed for energetic ions which adequately reproduces the experimental parameter dependence and allows extrapolation to ITER [2].

The present article will review the state of knowledge of chemical erosion and identify unresolved issues, such as the extrapolation of measured erosion yields at high particle fluxes towards low energies as expected in detached divertor plasmas and dependence of the composition of emitted molecules on ion energy. The understanding of transport and deposition of hydrocarbon molecules on the divertor plate requires sticking coefficients for different hydrocarbon radicals as well as re-erosion yields of deposited layers by atomic hydrogen.

\section{Status of knowledge of chemical erosion}

\subsection{Erosion by thermal atomic hydrogen $\mathrm{H}^{\mathbf{0}}$}

Chemical erosion of graphite due to thermal atomic hydrogen has been studied in great detail using a variety of diagnostics for the emitted species, the hydrogen content and the hybridization of carbon atoms in the surface layer [for example 3,4,5].

The individual steps in the erosion process have been elucidated and quantitatively described by cross sections or activation energies [1]. Hydrogen atoms are successively adsorbed to carbon atoms at the edges of graphitic planes until all carbon bonds are saturated and the surface is in a $\mathrm{sp}^{3}$ - hybridization state. At room temperature no erosion occurs. As the 
temperature increases hydrocarbon radicals in the neighborhood of unoccupied sites can be split-off thermally by simultaneously reforming of the graphitic double bond, thus returning to an $\mathrm{sp}^{2}$ hybridization. As the temperature increases further, adsorbed hydrogen can be released thermally before the surface is fully hydrogenated, thus preventing erosion. These combined processes are responsible for the observed temperature maximum in the erosion by thermal hydrogen atoms.

For thermal atoms the maximum yield varies from $10^{-4}$ to $10^{-1}$ from well annealed graphite to re-deposited layers. The erosion yield of a-C:H layers is strongly dependent on the structure of the layers at the surface [6]. The structure of a-C:H layers is mostly determined by their initial H content [7]. The released species for graphite are predominantly saturated heavier hydrocarbons, such as $\mathrm{C}_{2} \mathrm{H}_{4}$ and $\mathrm{C}_{3} \mathrm{H}_{6}$ molecules [8,9], with a large contribution of methane while the dominant species emitted from a-C:H layers are $\mathrm{CH}_{3}$ radicals [8].

\subsection{Chemical sputtering due to $\mathrm{H}^{0}$ and noble gas ions}

The erosion due to thermal atomic hydrogen can be enhanced by simultaneous impact of chemically inert ions, such as $\mathrm{Ar}^{+}$or $\mathrm{C}^{+}[10,11]$. This effect is named chemical sputtering and is the topic of the contribution by W. Jacob in this issue [12]. The enhancement may occur for two reasons: The simultaneous irradiation with energetic ions enhances the lattice disorder, thus creating new centers for adsorption of hydrogen. On the other hand, loosely bound hydrocarbon radicals which cannot be released at room temperature, may be released in kinetic bond breaking processes.

The first process leads to an increase of the chemical erosion at elevated temperatures, and eventually enhances the erosion yield of an impinging thermal hydrogen atom to the yield of energetic ions. The second process is most evident at room temperature, where no chemical erosion occurs without simultaneous ion bombardment. Both effects show a threshold related to the energy of the incident ions [13], and depend critically on the flux ratio of thermal hydrogen atoms to energetic ions. In contrast to chemical erosion at elevated temperatures the released species are predominantly $\mathrm{CH}_{3}$ radicals [8]. Details can be found in [12].

\subsection{Erosion due to energetic hydrogen ions $\mathrm{H}^{+}$}

Erosion yields and their energy dependence:

The bombardment of graphites by energetic hydrogen ions combines both the effects of energetic ions and hydrogen atoms thermalized at the end of the ion range inside the material. 
Actually, in graphite marked with a ${ }^{13} \mathrm{C}$ surface layer, it could be shown that the thermal chemical erosion process originates from the end of ion range, in contrast to physical sputtering [14].

In addition, chemical sputtering proceeds at room temperatures, with yield values of the order of $3 \%$ at energies where physical sputtering has disappeared due to a threshold energy for this process of about $30 \mathrm{eV}$. However, chemical sputtering for hydrogen ions also decreases towards a threshold energy and modeling of the bond breaking process by MD simulations quantify this threshold to about $2 \mathrm{eV}$ [15]. Recently, Hopf and Jacob [16] proposed an analytic model for the energy dependence of chemical sputtering of carbon by hydrogen isotope ions. They concluded that the threshold energy for this process should be in the range from 3 to 7 eV. Figure 1 shows the combined energy dependence of physical sputtering, chemical erosion and chemical sputtering together with an analytic description developed on the basis of the results by Küppers et al. [5] for thermal hydrogen atoms and the inclusion of damage production and chemical sputtering by energetic ions $[13,17]$. The analytic description adequately describes the chemical erosion in its energy and temperature dependence and can be used for extrapolations to divertor conditions in ITER [2]. The high values assumed for chemical erosion at $\mathrm{T}_{\max }$ down to below $1 \mathrm{eV}$ take into account the erosion due to thermal hydrogen atoms on a-C:H layers [18] while this erosion is negligible at room temperature.

\section{Diffusional effects at high ion energy:}

The fact that chemical erosion occurs at the end of the ion range requires that volatile reaction products diffuse to the surface, either through the crystalline lattice or along grain boundaries. In fine grain graphites or CFC materials porosity may facilitate diffusion. The diffusion of reaction products has been inferred from transient effects after rapidly switching on or off the ion beam at constant surface temperature $[19,20,21]$. When the beam is switched off, a sudden increase of the emission of hydrocarbons occurs before the mass spectroscopic signal falls off. In contrast, when the beam is switched back on during the decrease of the signal a transient drop of the signal is observed before the steady state emission is reestablished [19]. The transients are the more pronounced the higher the ion energy between $8 \mathrm{keV} \mathrm{D}{ }^{+}$and $100 \mathrm{keV}$ $\mathrm{D}^{+}$. These effects have been interpreted as the decomposition of reaction products by the incident ions during their diffusion to the surface. From more detailed studies a multi-region model for intra-granular diffusion and diffusion along grain boundaries was developed [20,21]. Similar transients occur upon rapid changes in temperature and ion flux [22]. The 
study and interpretation of these transients can give more detailed insight into the chemical erosion process, but has not yet been fully exploited.

Flux dependence of chemical erosion:

For the review on chemical erosion in 1998, the data situation at high ion fluxes did not allow a distinction between different models resulting in a weak or strong flux dependence, respectively [17]. A conservative recommendation for modeling assumed, therefore, no flux dependence [23]. Since 1998 several new results have been published [24,25] and reevaluated together with the set of previous data. After this re-evaluation and normalization of the data to one common ion energy, the following set of high flux data for methane production at $\mathrm{T}_{\max }$ is available (see Figure 2). The data are for $\mathrm{D}$ ions, normalized to an incident ion energy of $30 \mathrm{eV}$ and taken at or near $\mathrm{T}_{\max }$. While individual data sets in a narrow range of fluxes cannot clearly distinguish flux dependencies (PISCES, JET, JT-60U) the ensemble of data points and the individual investigations of PSI-1, TEXTOR and Tore Supra, spanning flux ranges of more than an order of magnitude, suggest a decrease of the erosion yield with ion flux, $\Phi$, starting at fluxes of about $10^{21} \mathrm{~m}^{-2} \mathrm{~s}^{-1}$.

As all investigators also have provided error bars for their yield values, a fit to the data using Bayesian probability theory has been made taking these errors into account [26,27]. This resulted in a decrease of the yield at high fluxes according to

$$
Y(E, T, \Phi)=\frac{Y_{\text {low }}(E, T)}{1+\left(\frac{\Phi}{6 \times 10^{21}}\right)^{0.54}}
$$

equ. 1

The exponent at high ion fluxes was determined to $0.54 \pm 0.04$. The same flux dependence, as given in equ. 1 for the erosion yield at $T_{\max }$ applies at room temperature (see results from ASDEX Upgrade [28]). Although there are no data at intermediate temperatures, it was assumed [27] that at all temperatures the same factor applies to the analytical description of chemical erosion, $\mathrm{Y}_{\text {low }}(\mathrm{E}, \mathrm{T})$, as given previously [17]. With this flux dependence, a description is now available which covers the energy, temperature and flux dependence adequately for extrapolation to wall and divertor conditions in ITER.

The common requisites for both erosion processes, the thermal chemical erosion and chemical sputtering at room temperature is the hydrogenation of surface carbon atoms to weakly bound hydrocarbon radicals. The fact that both erosion processes depend on ion flux in a similar manner indicates that the flux dependent reaction step lies in the sequential adsorption of hydrogen atoms. As full hydrogenation requires bond breaking processes and a change of 
carbon hybridization it is conceivable that individual steps are time dependent leading to the observed flux dependence. First MD simulations have indicated a supersaturation with hydrogen in the surface layer slowing down the erosion process [29]. To elucidate this effect in more detail further modeling is needed.

\section{Emitted species and their sticking coefficient}

\subsection{Emitted hydrocarbon molecules}

As mentioned above, for thermal atomic hydrogen impact the released species are predominantly saturated hydrocarbons, such as $\mathrm{CH}_{4}, \mathrm{C}_{2} \mathrm{H}_{\mathrm{x}}$ and $\mathrm{C}_{3} \mathrm{H}_{\mathrm{y}}$. For ion bombardment with energies above $10 \mathrm{eV}$ similar knowledge on the saturated hydrocarbons was collected [9,

30]. Emitted hydrocarbon molecules are not only $\mathrm{CH}_{4}$ molecules, but with about equal importance heavier hydrocarbons and radicals. The dependence on hydrogen isotope on the composition of emitted hydrocarbons is weak [31]. Very pronounced is the dependence on ion energy, with heavier hydrocarbons dominating at energies of $10 \mathrm{eV}$ and below, while at higher energies methane is the dominating reaction product (Figure 3). Table 1 gives values for deuterium bombardment of graphite collected by the group at University of Toronto.

As most of these measurements have been performed by residual gas analysis without direct sight to the target, only stable hydrocarbons could be detected, while radicals may be deposited to the vessel walls before reaching the mass analyzer.

\subsection{Emitted radicals}

Radicals can only be detected in direct line of sight to the target. Comparing the deposition on open collectors [32] or cavity probes [33] to total weight loss and residual gas measurements it was shown that typically $50 \%$ of the emitted species is deposited before reaching a remote mass spectrometer.

For the case of thermal hydrogen impact on a-C:H films typical mass spectra were reported by Vietzke [4]. He used a line-of-sight technique, which enables the mass analyzer to detect radicals without collision with the vessel walls (Figure 4). From a decomposition of the obtained mass spectra assuming stable hydrocarbons it is clear that additionally strong contributions from radical, especially $\mathrm{CH}_{3}, \mathrm{C}_{2} \mathrm{H}_{3}, \mathrm{C}_{3} \mathrm{H}_{3}$ and $\mathrm{C}_{3} \mathrm{H}_{5}$ have to be considered (Figure 4). The overall erosion yield of the a-C-H film is much larger than the erosion of graphite, actually close to the yield for energetic ions. Both findings demonstrate that 
chemical erosion of a-C:H layers proceeds similar to the case of chemical sputtering in the combination of thermal atomic hydrogen and energetic inert gas ions.

\subsection{Measurement of sticking coefficients}

As mentioned above, sticking of eroded species on the vessel walls or on the target influences weight loss and mass spectroscopy. In addition, it is of great importance in lifetime estimation of plasma facing components where in general re-deposition efficiencies of $90 \%$ or more are assumed. The transport of carbon containing species and final co-deposition with hydrogen isotopes constitutes the major source of the $\mathrm{T}$ inventory in a future fusion reactor. Emitted species include hydrocarbon radicals and sticking to chamber surfaces may be the reason for discrepancies between mass spectroscopy in the residual gas and weight loss experiments [32].

Deposited species in plasma experiments will be essentially the chemically emitted species and their fragments after dissociation in the boundary plasma [34,35]. They may be deposited as neutrals with just the energy received during break up or as ions accelerated in the sheath potential in front of the surface. The majority will have energies between 1 and $10 \mathrm{eV}$ [36] with a high probability of surviving the impact with surface atoms without break-up.

Recent data for the upper limit of the sticking coefficients [37] for a number of investigated hydrocarbons and radicals from a low temperature plasma [33,38,39] are compared in Table 2 with assumptions in current re-deposition modeling using MD simulations [40] (Figure 5).

These data need to be completed especially for heavier hydrocarbons. It needs to be clarified at which energy the sticking increases to higher values such as found in ion beam experiments [41]. However, at these energies low effective sticking coefficients are deduced from gas puff experiments through the TEXTOR limiter using ${ }^{13} \mathrm{CH}_{4}$ where surface analysis of the limiter showed very low net re-deposition of ${ }^{13} \mathrm{C}$ [42]. The very low effective sticking coefficients are assumed to result from efficient re-erosion of the deposited a-C:D layer due to synergistic erosion of thermal hydrogen and energetic ions [42]. These values should help in resolving the unpredicted carbon transport in the JET divertor, and are an indispensable pre-requisite for reliable extrapolation to ITER.

Sticking coefficients as well as the stability of the re-deposited layer are critically dependent on surface temperature. For $\mathrm{C}, \mathrm{C}_{2}$ and $\mathrm{C}_{3}$ a decrease of the sticking coefficient with increasing 
temperature has been observed [43]. At temperatures above $350^{\circ} \mathrm{C}$ no deposition was found for thermal $\mathrm{CH}_{3}$ radicals [44]. In the divertor chamber of ASDEX Upgrade the deposition decreases by a factor of 200 in going from room temperature to $250^{\circ} \mathrm{C}$ [45]. In order to control the T inventory in co-deposited layers it was proposed to keep divertor surfaces and pumping ducts above this temperature and collect carbon and tritium on specially equipped cooled surfaces [46].

\section{Unresolved issues for understanding and the extrapolation to ITER}

- How are hydrocarbons released from end of ion range? Are diffusional and break-up processes of reaction products involved?

- What is the origin of the composition of hydrocarbons and its change with ion energy?

- What is the origin of the flux dependence? Are individual steps with time duration of the order of 0.1-1 ms necessary?

- Can the threshold energy for chemical sputtering be confirmed experimentally? Can we model the CFC erosion in detached divertor conditions and extrapolate to ITER?

- More information is needed on sticking coefficients of radicals.

- More information is needed on the re-erosion of deposited a-C:H layers.

\section{Conclusions:}

Chemical erosion as dependent on ion energy, temperature and ion flux is reasonably well understood and analytically described. There is a gap in the experimental data between $10 \mathrm{eV}$ and thermal energies which needs to be closed by dedicated experiments and with the use of MD simulations in order to extrapolate to the detached divertor conditions in ITER. The composition of emitted species is important for further transport studies in the edge and divertor plasma of tokamaks and needs, therefore, further investigations.

Sticking coefficients for light hydrocarbon radicals may be much smaller than assumed in present modeling, while they may approach unity for heavier hydrocarbon radicals. As a rule of thumb the sticking coefficient depends on the carbon hybridization with values of $0.01,0.3$ and 1 for $\mathrm{sp}^{3}, \mathrm{sp}^{2}$ and $\mathrm{sp}^{1}$, respectively. These new data need to be completed and implemented in modeling codes. The dependence on radical energy between 1 and $10 \mathrm{eV}$ needs to be investigated theoretically (Molecular Dynamics calculations) and experimentally. 


\begin{tabular}{|c|c|c|c|c|c|}
\hline Hydrocarbons & $<1 \mathrm{eV}$ & $10 \mathrm{eV}$ & $50 \mathrm{eV}$ & $100 \mathrm{eV}$ & $200 \mathrm{eV}$ \\
\hline $\mathrm{CD}_{4}$ & $5 \%$ & $33 \%$ & $45 \%$ & $63 \%$ & $73 \%$ \\
\hline $\mathrm{C}_{2} \mathrm{D}_{\mathrm{x}}$ & $50 \%$ & $42 \%$ & $35 \%$ & $21 \%$ & $19 \%$ \\
\hline $\mathrm{C}_{3} \mathrm{D}_{\mathrm{y}}$ & $45 \%$ & $25 \%$ & $20 \%$ & $16 \%$ & $8 \%$ \\
\hline
\end{tabular}

\begin{tabular}{|l|l|l|}
\hline Molecule & $\begin{array}{l}\text { Experimental } \\
\text { for } 1100 \mathrm{~K} \\
{[6]}\end{array}$ & $\begin{array}{l}0.02 \text { to } \\
10 \mathrm{eV} \\
{[40]}\end{array}$ \\
\hline $\mathrm{CH}_{4}$ & 0 & n.a. \\
\hline $\mathrm{CH}_{3}$ & $10^{-4}$ to $10^{-2}$ & 0.02 to 1 \\
\hline $\mathrm{CH}_{2}$ & 0.025 & 0.03 to 1 \\
\hline $\mathrm{CH}^{-}$ & 1 & n.a \\
\hline $\mathrm{C}$ & 1 & 1 \\
\hline $\mathrm{C}_{2} \mathrm{H}$ & 0.8 & 0.15 to 1 \\
\hline $\mathrm{C}_{2} \mathrm{H}_{3}$ & 0.35 & 0.02 to 1 \\
\hline $\mathrm{C}_{2} \mathrm{H}_{5}$ & 0.03 & n.a. \\
\hline Table 2: Comparison of experimental \\
and calculated sticking \\
coefficients
\end{tabular}




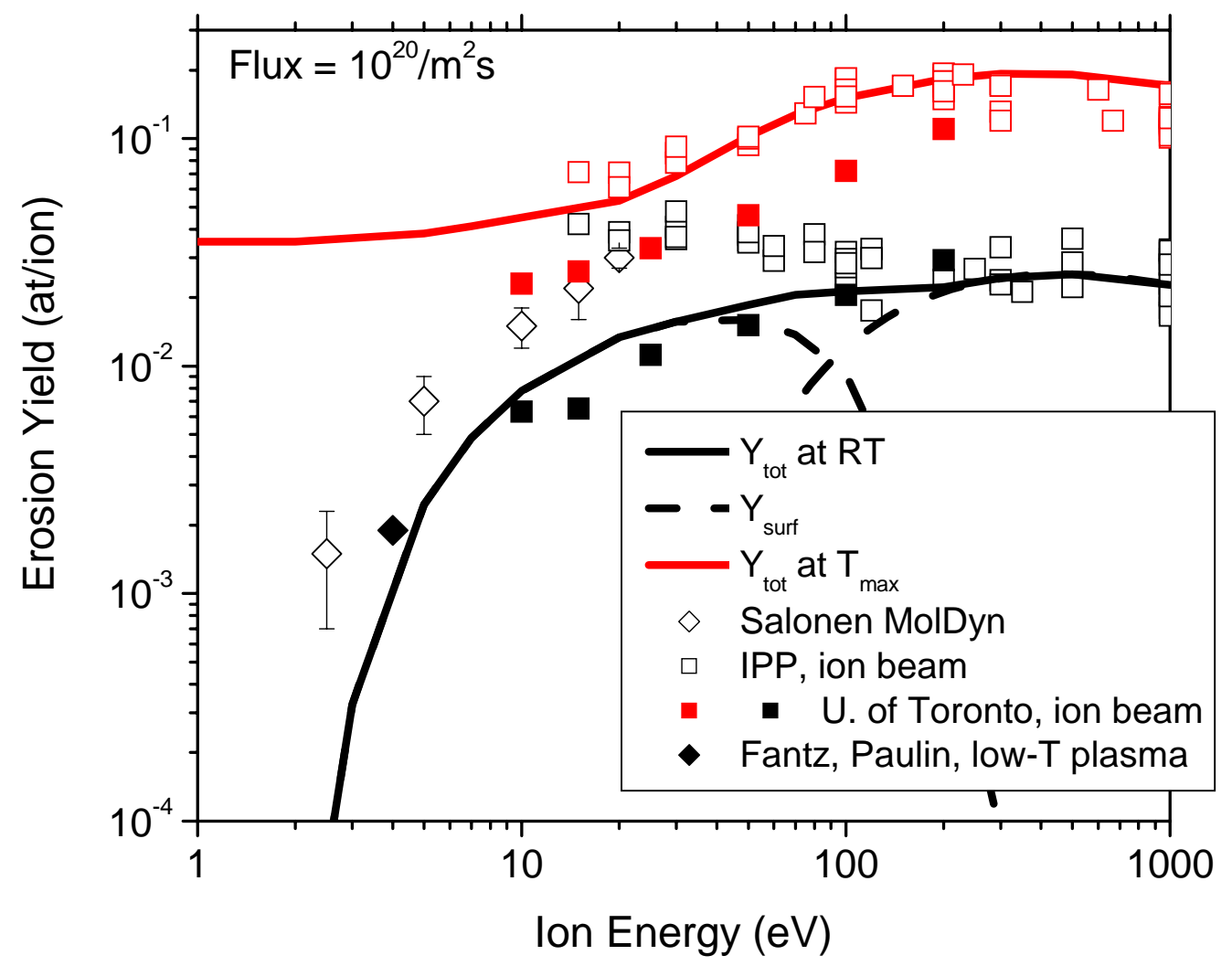

Figure 1: Energy dependence of chemical erosion at $T_{\max }$ and chemical sputtering at room temperatures for deuterium ions. Note that indications for a threshold for chemical erosion are based on molecular dynamics modeling. 


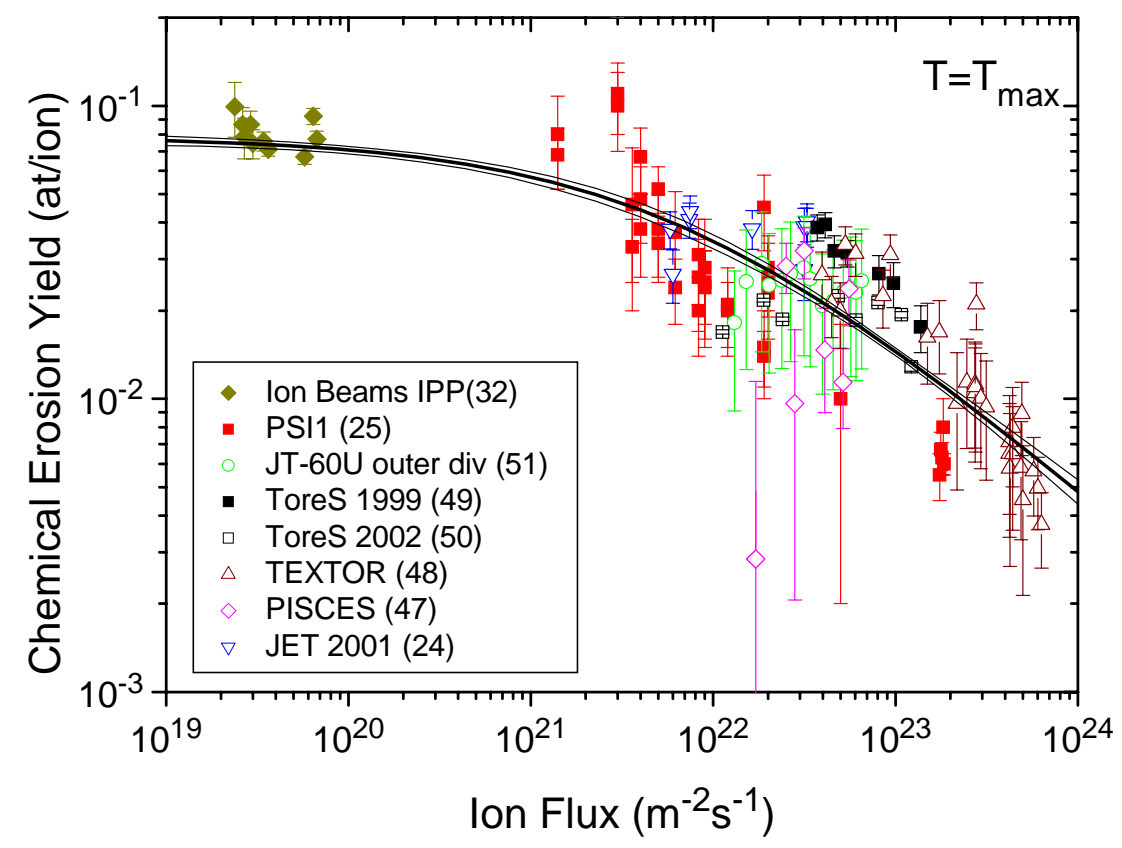

Figure 2: Flux dependence of chemical erosion at $\mathrm{T}_{\max }$ as measured in ion beam experiments [32], plasma simulators (PSI1 [25], PISCES [47]) and fusion limiter (TEXTOR [48], Tore Supra [49,50]) and divertor (JET [24], JT-60U [51]) experiments [details see ref 27]. 


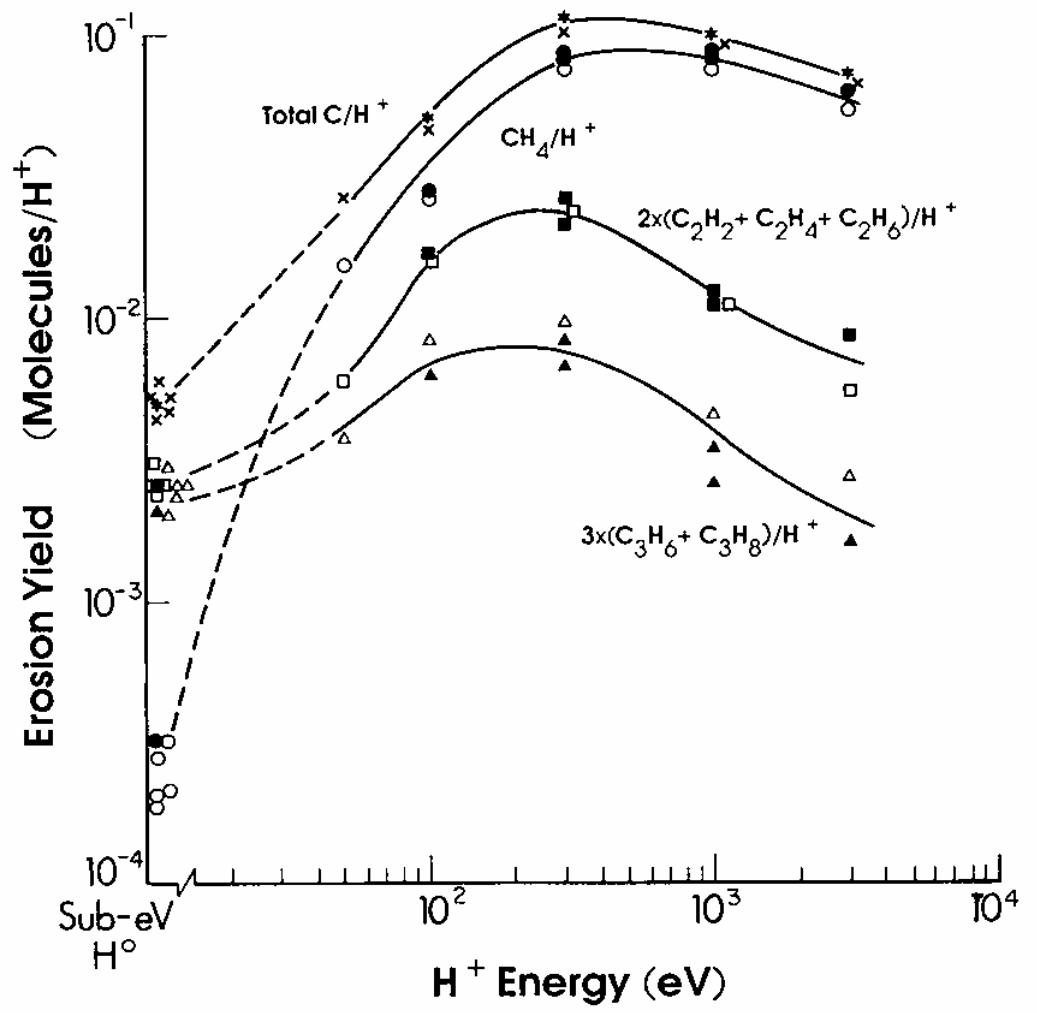

Figure 3: Composition of emitted saturated hydrocarbons at $\mathrm{T}_{\max }$ as function of particle energy [9]. 


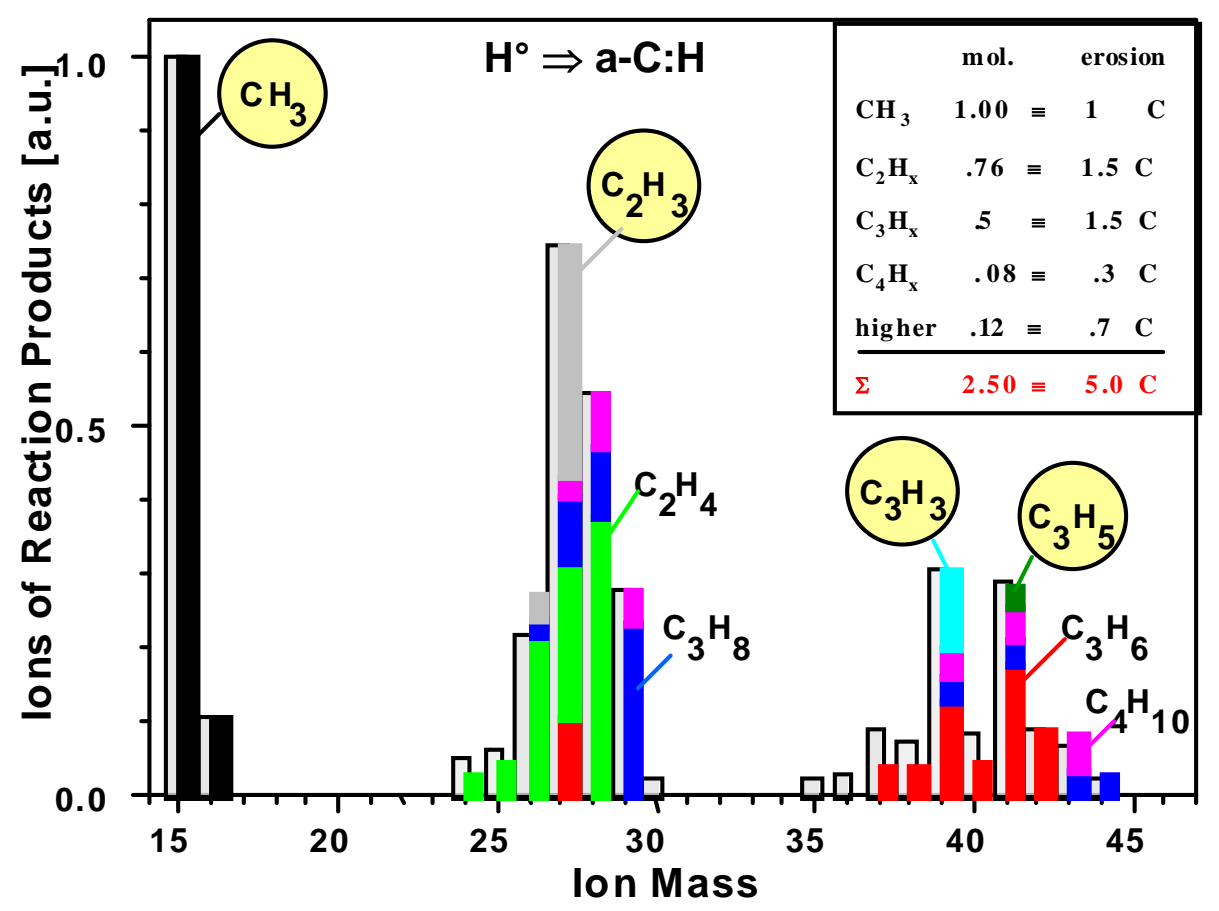

Figure 4: Composition of reaction products determined in line-of-sight mass spectrometry demonstration the importance of radical emission, such as $\mathrm{CH}_{3}, \mathrm{C}_{2} \mathrm{H}_{3}, \mathrm{C}_{3} \mathrm{H}_{3}$ and $\mathrm{C}_{3} \mathrm{H}_{5}$ [4]. 


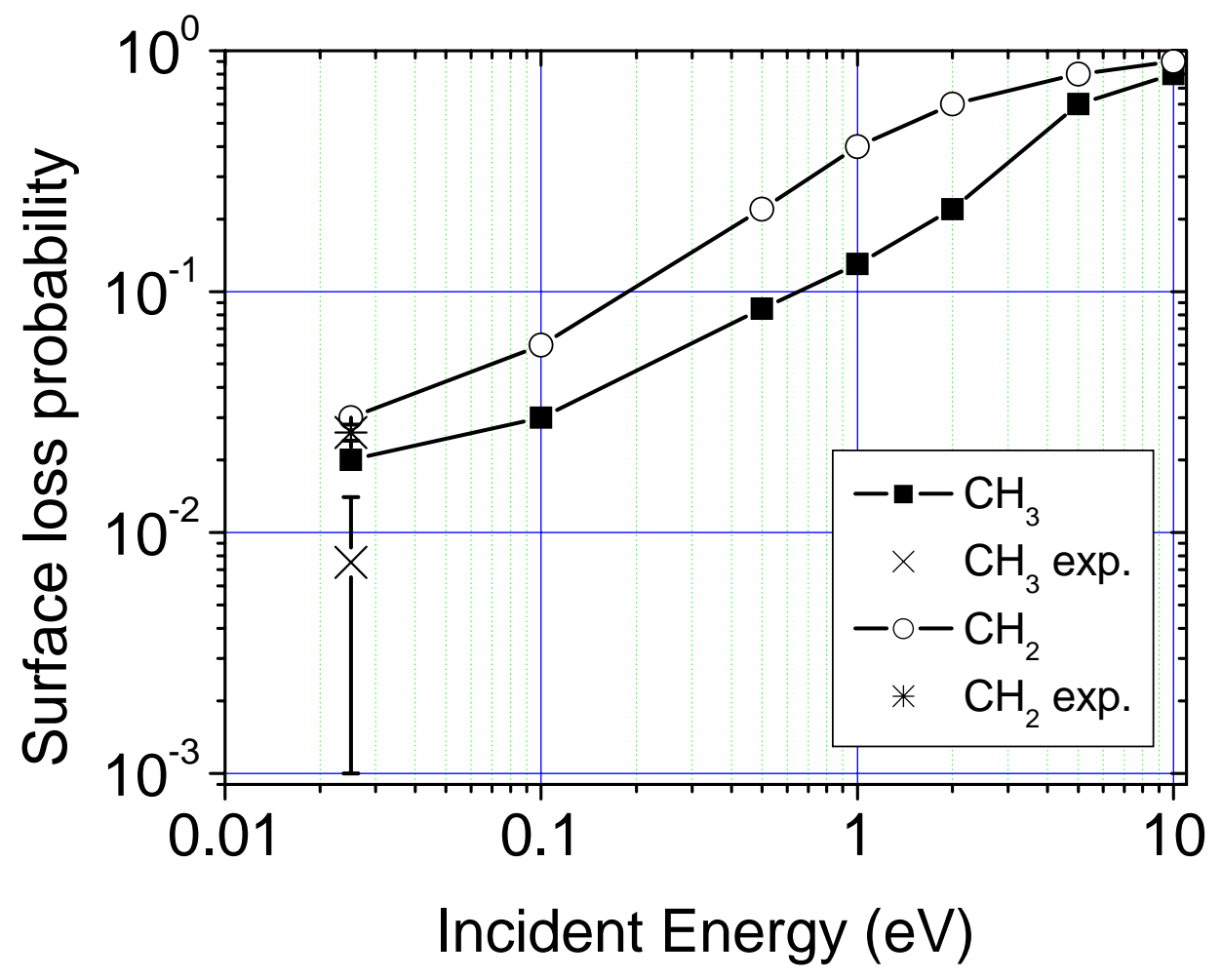

Figure 5: Comparison of measured [39] with calculated sticking coefficients using molecular dynamics simulation as function of particle energy [40]. 


\section{References:}

[1] A. Horn, A. Schenk, J. Biener, B. Winter, C. Lutterloh, M. Wittmann, J. Küppers, Chem. Phys. Lett. 231 (1994) 193

[2] J. Roth, A. Kirschner et al. J. Nucl. Mater. 337-339 (2005) 970

[3] J. W. Davis, A.A. Haasz, P.C. Stangeby, J. Nucl. Mater. 155-157 (1988) 234

[4] E. Vietzke, K. Flaskamp, V. Philipps, G. Esser, P. Wienhold, J. Winter, J. Nucl. Mater. 145-147 (1987) 443

[5] M. Wittmann, J. Küppers, J. Nucl. Mater. 227 (1996) 186

[6] W. Jacob, J. Nucl. Mater. 337-339 (2005) 839

[7] T. Schwarz-Selinger, A. von Keudell, W. Jacob, J. Applied Physics 86, 3988 (1999).

[8] E. Vietzke, K. Flaskamp, V. Philipps, J. Nucl. Mater. 128\&129 (1984) 545

[9] E. Vietzke, A.A. Haasz, "Chemical Erosion" in "Physical Processes of the Interaction of Fusion Plasmas with Solids”, eds. W. Hofer, J. Roth (Academic Press, San Diego 1996), p. 135

[10] E. Vietzke, K. Flaskamp, V. Philipps, J. Nucl. Mater. 111-112 (1982) 763

[11] J. Davis, A.A. Haasz, Appl. Phys. Lett. 57 (1990) 1976

[12] W. Jacob, these proceedings

[13] J. Roth, C. Garcia-Rosales, Nucl. Fusion 36 (1996) 1647 with corrigendum Nucl. Fusion 37 (1997) 897

[14] J. Roth, J. Bohdansky, Appl. Physics Letters 51 (1987) 964

[15] E. Salonen, K. Nordlund, J. Keinonen and C. Wu, Phys. Rev. B 63 (2001) 195415

[16] C. Hopf, W. Jacob, J. Nucl. Mater, 342 (2005) 141

[17] J. Roth, J.Nucl. Mater. 266-269 (1999) 51

[18] E. Vietzke, V. Philipps, Fusion Technology 15 (1989) 108

[19] P. Franzen, Max-Planck-Institut für Plasmaphysik, Report IPP 9/92 (1993)

[20] A.A. Haasz et al, J.Nucl. Mater. 220-222 (1995) 815

[21] S. Chiu et al., J. Nucl. Mater. 218 (1995) 319

[22] J. Roth, J. Bohdansky, K.L. Wilson, J. Nucl. Mater. 111-112 (1982) 775

[23] J.N. Brooks, G. Federici, D.N. Ruzic, D.G. Whyte, J. Nucl. Mater. 266-269 (1999) 58

[24] M.F. Stamp, S.K. Erents, W. Fundamenski, G.F. Matthews, R.D. Monk, Physica Scripta T91 (2001) 13

[25] P. Kornejew, W. Bohmeyer, H.-D. Reiner, C.H. Wu, Physica Scripta T91 (2001) 29

[26] V. Dose, J. Roth, R. Preuss, J. Nucl. Mater 288 (2001) 153

[27] J. Roth, R. Preuss et al., Nuclear Fusion 44 (2004) L21

[28] R. Pugno et al., J. Nucl. Mater. 337-339 (2005) 985

[29] E. Salonen, K. Nordlund, J. Keinonen and C. H. Wu, Journal of Nuclear Materials 290-293 (2001) 144

[30] J. Roth, E. Vietzke, A.A. Haasz, in: Atomic and Plasma-Material Interaction Data for Fusion, Suppl. to Nuclear Fusion, Vol. 1, IAEA Vienna (1991)

[31] B.V. Mech, A.A. Haasz, J.W. Davis, J. Nucl. Mater. 255 (1998) 153

[32] M. Balden, J. Roth, J. Nucl. Mater. 280 (2000) 39

[33] C. Hopf, T. Schwarz-Selinger, W. Jacob, W., A. von Keudell, J. Appl. Physics 87, 2719 (2000)

[34] J. N. Brooks, et al., J. Nucl. Mater. 241-243, 294 (1997)

[35] J. N. Brooks, et al. J. Nucl. Mater. 266-269, 58 (1999) 
[36] J. Brooks, (2000) private communication

[37] C. Hopf, T. Schwarz-Seliger, W. Jacob, A. von Keudell, J. Appl. Phys. 87 (2000) 2719

[38] H. Toyoda, et al., Phys. Lett. 54, 1507 (1989)

[39] A. von Keudell, T. Schwarz-Selinger, M. Meier, W. Jacob, Appl. Phys. Letters 76, 676 (2000) with corrigendum Appl. Phys. Letters 77, 459 (2000)

[40] D. A. Alman, D. N. Ruzic, Physica Scripta T111 (2004) 145

[41] W. Wang, et al., Nuclear Instr. and Meth. B129, 210 (1997)

[42] A. Kirschner, V. Philipps, J. Winter, U. Kögler, Nuclear Fusion 40 (2000), 989

[43] V. Philipps, E. Vietzke, K. Flaskamp, Surf. Science 178, 806 (1986)

[44] M. Meier, A. von Keudell, J. Chem. Phys. 116 (2002) 5125

[45] M. Mayer et al., (2004) private communication

[46] A. von Keudell, C. Hopf, T. Schwarz-Seliger, W. Jacob, Nuclear Fusion 39 (1999) 1451

[47] D.G. Whyte et al., Nuclear Fusion 41 (2001) 47

[48] A. Pospieszczyk et al., Report UCLA-PPG-1251 (1989)

[49] R. Ruggieri et al., J. Nucl. Mater. 266-269 (1999) 660

[50] A. Cambe et al., J. Nucl. Mater. 313-316 (2003) 364

[51] T. Nakano et al., Nuclear Fusion 42 (2002) 689 